\title{
Large impact velocities suppress the splashing of micron-sized droplets
}

\author{
Masashi Usawa, ${ }^{1}$ Yuta Fujita, ${ }^{1}$ Yoshiyuki Tagawa $\odot,{ }^{1, *}$ \\ Guillaume Riboux $\odot{ }^{2}$ and José Manuel Gordillo ${ }^{2}{ }^{2, \dagger}$ \\ ${ }^{1}$ Department of Mechanical Systems Engineering, Tokyo University of Agriculture and Technology, \\ 1848588 Tokyo, Japan \\ ${ }^{2}$ Área de Mecánica de Fluidos, Departamento de Ingeniería Aeroespacial y Mecánica de Fluidos, \\ Escuela Superior de Ingenieros, Universidad de Sevilla, 41092 Sevilla, Spain
}

(Received 6 October 2020; accepted 26 January 2021; published 15 February 2021)

\begin{abstract}
Here we investigate the transition from spreading to splashing of drops with radii $R$ varying from millimeters to tens of microns impacting onto a smooth and dry partially wetting substrate at normal atmospheric conditions. Experiments show that the smaller $R$ is, the larger the impact velocity $V$ for the drop to splash needs to be but also that splash is inhibited if $\mathrm{We}_{\lambda}=\rho V^{2} \lambda / \sigma \gtrsim 0.5$, with $\sigma, \rho$, and $\lambda$ indicating the interfacial tension coefficient, the liquid density, and the mean free path of gas molecules. This result has been validated for two different values of the Ohnesorge number $\mathrm{Oh}_{\lambda}=\mu / \sqrt{\rho \lambda \sigma}$, with $\mu$ indicating the liquid viscosity, defined using only the material properties of the liquid and of the surrounding gaseous atmosphere. The underlying reason for this a priori unexpected finding results from the fact that the thin liquid film ejected after the drop touches the substrate is, under many practical conditions, $H_{t} \simeq \sigma /\left(\rho V^{2}\right)$ Riboux and Gordillo [Phys. Rev. Lett. 113, 024507 (2014)]. Then, for sufficiently large values of $V$, the thickness of the lamella becomes similar to the mean free path of gas molecules, i.e., $H_{t} \approx \lambda$, and, under these conditions, the splash of the drop is inhibited because the lift force causing the liquid to dewet the partially wetting solid is negligible. The spreading to splashing and the splashing to spreading transitions observed experimentally as the impact velocity is increased and the radii of the droplets is above a certain threshold value are very well predicted by the theory in G. Riboux and J. M. Gordillo [Phys. Rev. Lett. 113, 024507 (2014)] and J. M. Gordillo and G. Riboux [J. Fluid Mech. 871, R3 (2019)] once the aerodynamic lift force is set to zero for $H_{t} / \lambda \lesssim 2$, i.e., when $\mathrm{We}_{\lambda} \gtrsim 0.5$.
\end{abstract}

DOI: 10.1103/PhysRevFluids.6.023605

\section{INTRODUCTION}

Ink-jet printing is based on the fact that an initially spherical droplet impacting against a solid deforms and creates, after drying, a dot fixed on the substrate [1]. The contour of the image resulting when a number of properly placed dots gather together is smooth if the perimeter of a single dot is circular, which happens if the drop spreads over the substrate [2-7] or, in contrast, will be irregular if the drop splashes [8] (see Fig. 1). It is known that the spreading-splashing transition takes place,

\footnotetext{
*tagawayo@cc.tuat.ac.jp

$\dagger$ jgordill@us.es
}

Published by the American Physical Society under the terms of the Creative Commons Attribution 4.0 International license. Further distribution of this work must maintain attribution to the author(s) and the published article's title, journal citation, and DOI. 


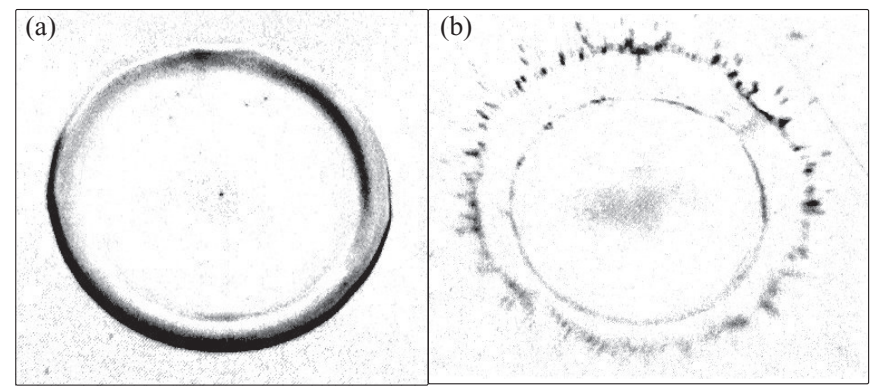

FIG. 1. The final shape of a impacting drop onto a smooth solid substrate is circular if the drop spreads over the solid (a) or it is star shaped if the drop splashes (b).

for given material properties of both the liquid and the surrounding gaseous atmosphere, when the impact velocity $V$ of a drop of radius $R$ is above the critical velocity for splashing, $V>V^{*}$, which is usually of a few meters per second [9-11]. Here we will demonstrate that there exists a second transition from splashing to spreading taking place for larger impact velocities of a few tens of meters per second like those found, for instance, in new printing applications. We will show that the reason behind this behavior relies on the fact that the thickness of the lamella ejected tangentially to the substrate can be similar to the mean free path of gas molecules for sufficiently large values of the drop impact velocity.

Notice that fast printing processes impose drop impact velocities such that $V \gtrsim 5 \mathrm{~m} / \mathrm{s}$ and that the usual spatial resolution requirements of the printed image demand that $R<10^{-3} \mathrm{~m}$. However, most of the experimental evidence in the recent literature, with the exception of the remarkable contributions by Visser et al. [5,6], who did not observe splash for high-speed micronsized water droplets, describes the spreading-splashing transition of millimetric drops. Hence, in spite of its clear interest for ink-jet printing applications, there is a lack of understanding of the precise conditions under which micron-sized droplets spread or splash after impacting a smooth and dry partially wetting substrate. The purpose of this contribution is to provide experimental evidence describing the spreading-splashing transition of micron-sized droplets impacting against a partially wetting substrate at normal atmospheric conditions. The experimental function $V^{*}(R)$ with $R$ varying within the range of sizes found in different technological applications, namely $1.5 \times 10^{-3} \mathrm{~m}<R \lesssim 45 \times 10^{-6} \mathrm{~m}$, will be compared with the predictions made by the theory in Refs. $[9,10,12]$, finding good agreement. The effects associated with the substrate temperature [13-16], surface texture, or wettability [17-21] will not be considered here and could be the subject of a future contribution.

\section{EXPERIMENTS}

In all experiments presented here, an ethanol drop of radius $R$, density $\rho=789 \mathrm{~kg} / \mathrm{m}^{3}$, dynamic viscosity $\mu=10^{-3} \mathrm{Pas}$ and interfacial tension coefficient $\sigma=2.2 \times 10^{-2} \mathrm{~N} / \mathrm{m}$, impacts with a velocity $V$ against a dry and smooth silicon wafer at normal atmospheric conditions; therefore, the values of the density, viscosity, and mean free path of the gas are respectively given by $\rho_{g} \simeq$ $1.2 \mathrm{~kg} / \mathrm{m}^{3}, \mu_{g}=1.8 \times 10^{-5} \mathrm{~Pa} \mathrm{~s}$, and $\lambda=6.9 \times 10^{-8} \mathrm{~m}$. Since the radii of the impacting drops is varied from $R \simeq 1.5 \times 10^{-3} \mathrm{~m}$ to $R \simeq 4.5 \times 10^{-5} \mathrm{~m}$, and the drop impact speed is varied between $V \simeq 1 \mathrm{~m} / \mathrm{s}$ and $V \simeq 18 \mathrm{~m} / \mathrm{s}$, three different types of experimental setups have been used: Figure 2 A(a) shows that millimetric drops are formed from a needle placed at a variable height from the impacting surface and Fig. 2A(b) shows that drops with radii varying between $8 \times 10^{-5} \mathrm{~m}$ and $3.5 \times 10^{-4} \mathrm{~m}$ are formed using the method described in Refs. [22,23], where a device produces a fast and highly stretched micron-sized jet as a consequence of the sudden acceleration of a capillary tube which is initially filled with a liquid that forms a concave interface with the surrounding air. 


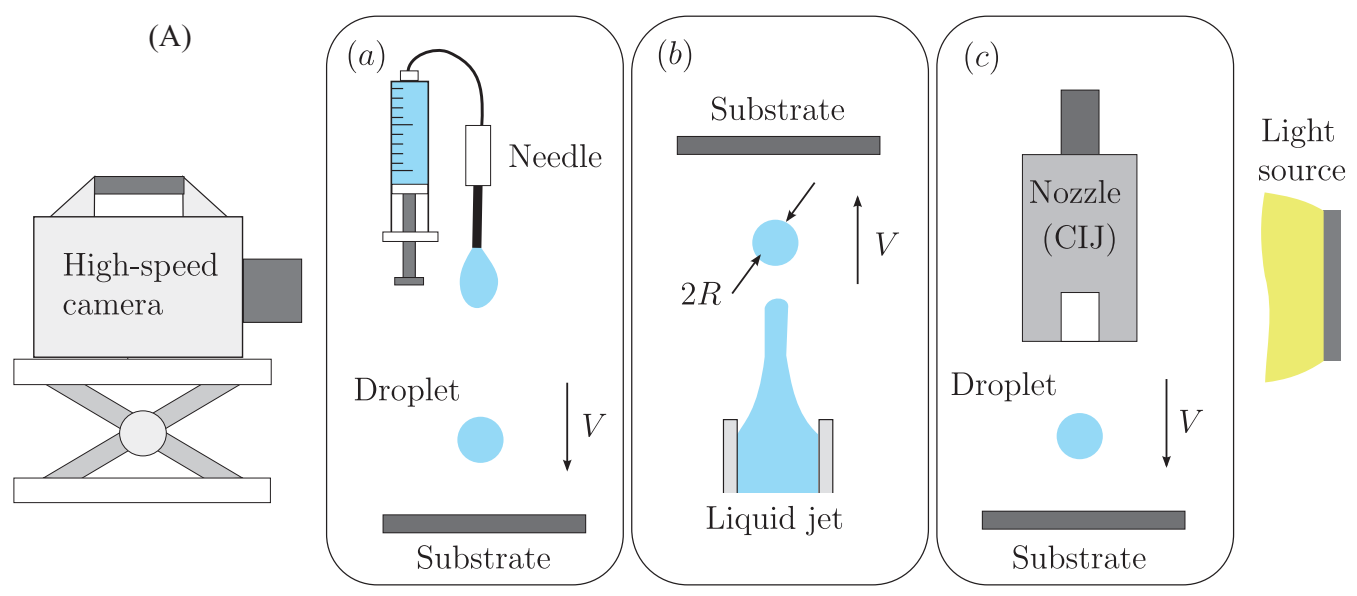

(B)

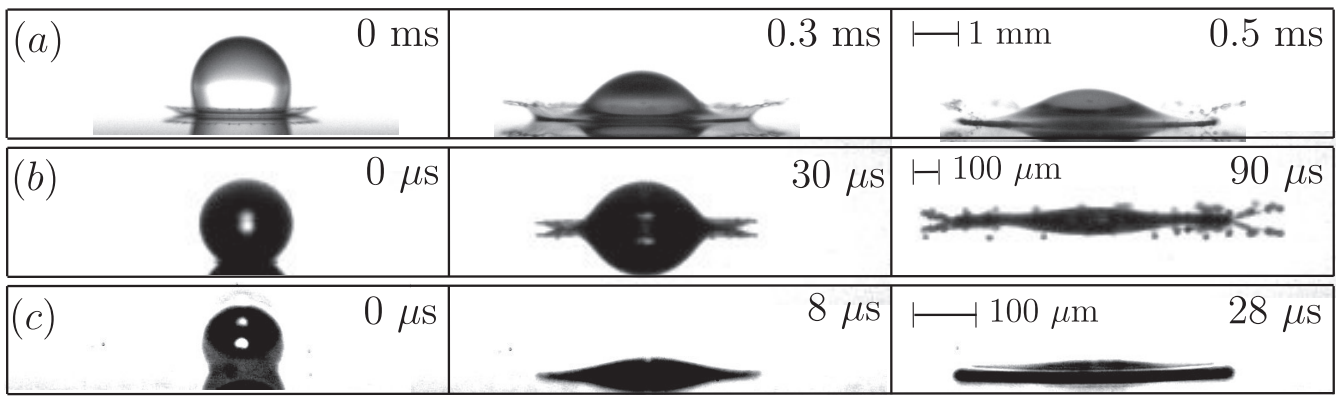

FIG. 2. (A) Sketch of the different experimental setups used to produce droplets with radii varying from millimeters to tens of microns. These droplets impact against a dry and smooth silicon substrate at normal atmospheric conditions. (a) Quasistatic generation of millimeter-sized drops, $R \sim 1.15 \mathrm{~mm}\left(\mathrm{Oh}=7 \times 10^{-3}\right)$, from a needle placed at different heights from the impacting surface in order to change the impact velocity, $V$, within the range $0.96<V<2.98 \mathrm{~m} / \mathrm{s}$. In this case, the high-speed camera (FASTCAM, SA-X, Photron) is operated at 10000 frames per second (f.p.s.) and the spatial resolution is $\sim 10 \mu \mathrm{m} /$ pix. (b) Generation of micron-sized droplets by means of the device described in Ref. [22]. The radii of the droplets produced in this way vary within the range $R \sim 79-330 \mu \mathrm{m}\left(\mathrm{Oh} \sim 13 \times 10^{-3}-27 \times 10^{-3}\right)$, whereas the impact velocity varies within the range $V \sim 1-16 \mathrm{~m} / \mathrm{s}$. The high-speed camera (FASTCAM, SA-X, Photron) is operated at 100000 f.p.s. and, in this case, the spatial resolution is $\sim 8 \mu \mathrm{m} /$ pix. (c) A continuous ink-jet printer (CIJ) (Kishu Giken), is used to produce droplets with radii $R \sim 45-69 \mu \mathrm{m}\left(\mathrm{Oh} \sim 29 \times 10^{-3}-36 \times 10^{-3}\right)$ and impact velocities vary within the range $V \sim 12.2-18.0 \mathrm{~m} / \mathrm{s}$. In this case, the drop impact process is recorded using a high-speed camera (Kirana, Nobby Tech) operated at 500000 f.p.s., obtaining images with a spatial resolution of $1.5 \mu \mathrm{m} /$ pix. (B) Images obtained using each of the three setups depicted in (A). (a) A drop of radius $R \sim 1.15 \mathrm{~mm}$ impacting at $V \sim 3 \mathrm{~m} / \mathrm{s}$ (We $\sim 365$ ) splashes and disintegrates into tiny droplets. (b) Splashing of a drop of radius $R \sim 200 \mu \mathrm{m}$ impacting at $V \sim 6 \mathrm{~m} / \mathrm{s}$ (We $\sim 254$ ). (c) Spreading of a drop of radius $R \sim 65 \mu \mathrm{m}$ impacting at $V \sim 18 \mathrm{~m} / \mathrm{s}$ (We $\sim 742$ ). The limited spatial resolution does not exclude the unlikely possibility that submicron-sized droplets are produced in the experiments.

Finally, Fig. 2A(c) shows that even smaller and faster droplets are produced using a continuous ink-jet printer (CIJ) (Kishu Giken). In this printing technology, a continuous micron-sized liquid jet breaks into droplets by exciting harmonically the imposed liquid flow rate. The experimental values of both $R$ and $V$, represented in Fig. 3 using either hollow or solid symbols to denote spreading or splashing events respectively, are extracted from the analysis of the videos recorded using two different types of high-speed cameras, see the caption in Fig. 2. 


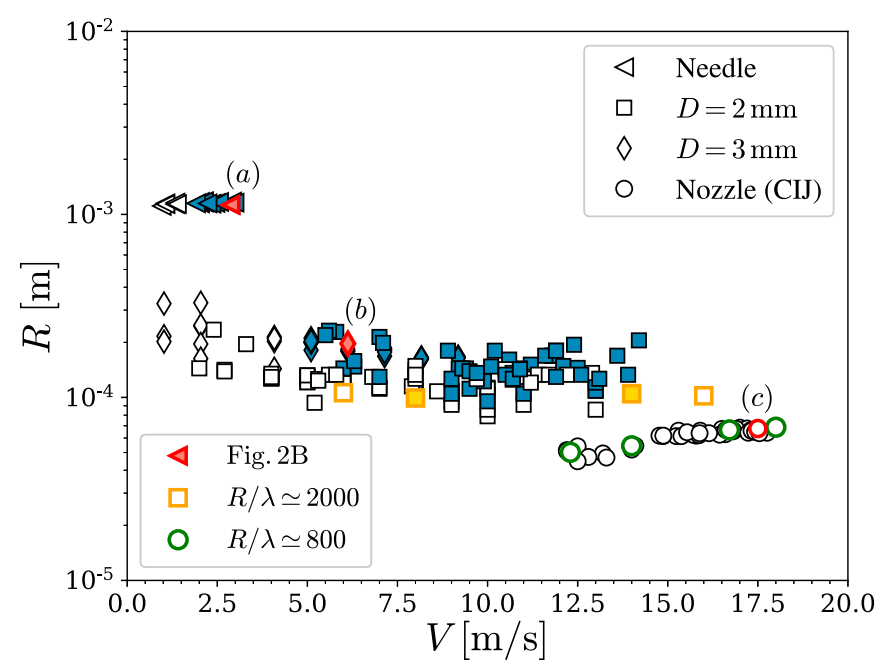

FIG. 3. This figure represents the range of experimental values of $R$ and $V$ investigated here for each of the three different experimental setups sketched in Fig. 2, with $D$ indicating the diameter of the capillary tube used in the device described in Ref. [22]. Empty symbols indicate that the droplet spreads over the substrate and blue ones that the droplet splashes. Orange and green symbols indicate the experimental conditions represented at the top and bottom rows of Fig. 4, respectively. The symbols in red indicate the values of $R$ and $V$ corresponding to the experimental images in Fig. 2(B).

The analysis of the experimental images at the top sequence of images in Fig. 4, which represent the effect of increasing the impact speed of droplets with radii $R / \lambda \approx 2000$ reveals that, analogously with the case of millimetric droplets, an increase of the impact velocity promotes a transition from spreading to splashing but also that splashing is suppressed when the impact velocity is further increased. Notice that the value of the impact velocity $V$ is represented in Fig. 4 in terms of the dimensionless parameter,

$$
\mathrm{We}_{\lambda}=\frac{\rho V^{2} \lambda}{\sigma}=\mathrm{We} \frac{\lambda}{R},
$$

with the Weber number We, the Ohnesorge Oh, and the Reynolds Re numbers defined here as

$$
\mathrm{We}=\frac{\rho V^{2} R}{\sigma}, \mathrm{Oh}=\frac{\mu}{\sqrt{\rho R \sigma}}, \mathrm{Re}=\frac{\sqrt{\mathrm{We}}}{\mathrm{Oh}} .
$$

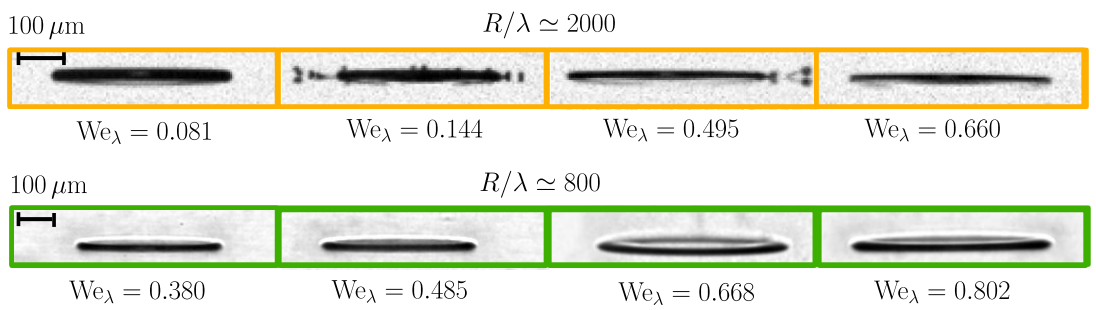

FIG. 4. The top sequence of images shows the effect of increasing the drop impact velocity for the case of droplets with radii $R / \lambda \approx 2000$, where a spreading-splashing-spreading transition is observed. The sequence at the bottom, where no splash is observed in spite of the impact velocities are even larger than those in the images at the top, corresponds to the case of drops with $R / \lambda \approx 800$ generated by means of a CIJ printer. The experimental conditions corresponding to the images at the top and bottom rows are indicated in Fig. 3 using, respectively, orange and green symbols. 


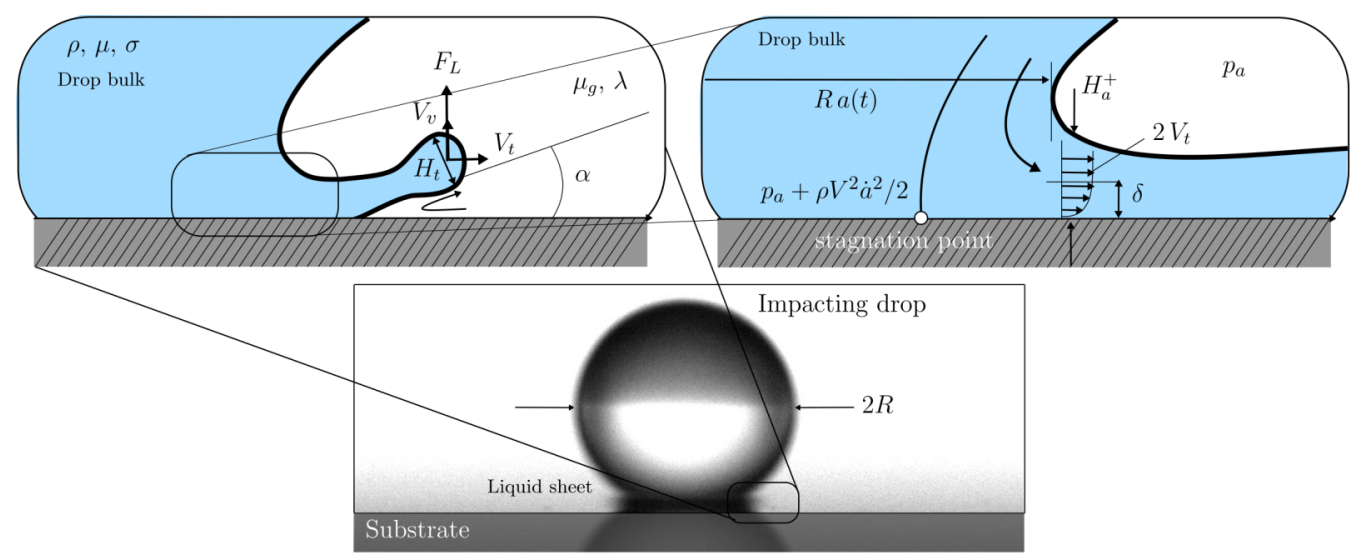

FIG. 5. Sketch and definition of the different variables used in the analysis in main text, with $F_{L}$ the lift force, $H_{t}$ and $V_{t}$ denoting the thickness and velocity of the edge of the expanding liquid sheet and $V_{v}$ the vertical velocity. All these variables are functions of the dimensionless ejection time $t_{e}=T_{e} V / R$, defined as the instant at which the thin liquid sheet is ejected tangentially to the substrate. The angle $\alpha$ refers to the wedge angle formed by the advancing rim and the substrate. The flow field entering into the lamella at the top right sketch is represented in a frame of reference moving with the velocity at which the drop wets the substrate, namely $V \dot{a}=V d a / d t$ with $a=\sqrt{3 t}$ and $t=V T / R$ the dimensionless time. The expression for the thickness of the boundary layer $\delta$ in Eq. (9) was deduced in Ref. [12] in the moving frame of reference, where there exists a stagnation point, also represented in the sketch.

The result shown at the bottom sequence of images in Fig. 4, which correspond to the case of drops with radii $R / \lambda \approx 800$, produced using a CIJ printer, is equally interesting because no splashing is observed, no matter how large the impact velocity is. The purpose of the next section will be to explain and quantify the experimental observations depicted in Figs. 3 and 4.

\section{THEORY}

In Refs. $[9,10,12]$ it was shown that, once a liquid sheet is expelled at a velocity $V_{t}>V$ after the drop touches the solid substrate, its edge experiences a vertical lift force per unit length $F_{L}$ caused by the gas lubrication layer located beneath the liquid rim, which induces a vertical velocity to the front of the expanding liquid sheet $V_{v} \propto \sqrt{F_{L} /\left(\rho H_{t}\right)}$, with $H_{t}$ indicating the rim thickness, see Fig. 5. The vertical velocity imparted to the edge of the expanding liquid sheet could make the lamella to take off from the substrate if this velocity is larger than the velocity of radial growth of the rim caused by capillary retraction, $\propto \sqrt{2 \sigma /\left(\rho H_{t}\right)}$. Hence, the critical velocity for splashing can be determined from the condition $\sqrt{F_{L} /(2 \sigma)}=\beta$ with $\beta$ a constant. In Refs. [9,10,12], the lift force $F_{L}$ was expressed as the addition of the lubrication force exerted by the gas flow at the wedge region formed by the advancing liquid sheet and the substrate, $K_{l} \mu_{g} V_{t}$, with $K_{l}$ a function depending on the ratio $H_{t} / \lambda$ and $V_{t}$ the relative velocity between the gas and the liquid, plus the aerodynamic force $K_{u} \rho_{g} V_{t}^{2} H_{t}$, with $K_{u}$ a constant that, thanks to the experimental results by Ref. [24], we approximated in Ref. [10] to $K_{u} \simeq 0$, see Fig. 5. These facts yield that the splash criterion in Refs. [9,12] can be written as it was proposed in Ref. [10]:

$$
\frac{K_{l} \mu_{g} V_{t}}{\sigma}=0.034
$$

with $V_{t}$ related with the instant $T_{e}$ the lamella is initially ejected tangentially to the wall as:

$$
V_{t}=V(\sqrt{3} / 2) t_{e}^{-1 / 2}
$$


where $t_{e}=T_{e} V / R$ refers to the dimensionless ejection time, which is calculated using the expression given in Ref. [9],

$$
\frac{\sqrt{3}}{2} \mathrm{Re}^{-1} t_{e}^{-1 / 2}+\mathrm{Re}^{-2} \mathrm{Oh}^{-2}=1.1 t_{e}^{3 / 2} .
$$

The expression for the coefficient $K_{l}$ in (3) varies depending on the relative values of the slip lengths at the solid wall and of the one induced by the shear stresses exerted by the gas flow at the air-liquid interface: Indeed, using the results in Ref. [10], if We $\lambda / R<\left(\mu_{g} / \mu\right)^{3 / 4} \mathrm{Oh}^{1 / 4}$, then

$$
K_{l} \simeq \frac{1}{2}\left(\frac{6}{\tan ^{2} \alpha}\right) \ln \left[0.011\left(\frac{\mu}{\mu_{g}}\right)^{3 / 4} \mathrm{Oh}^{-1 / 4}\left(\frac{H_{t}}{\lambda}\right)\right],
$$

and when We $\lambda / R>\left(\mu_{g} / \mu\right)^{3 / 4} \mathrm{Oh}^{1 / 4}$

$$
K_{l} \simeq\left(\frac{6}{\tan ^{2} \alpha}\right) \ln \left[1+\frac{H_{t}}{19 \lambda}\right]
$$

with $\alpha \simeq 60^{\circ}$ [9] the angle of the wedge formed by the lifting lamella and the solid substrate, see Fig. 5 .

Notice that the splash criterion in Eq. (3) crucially depends on the ratio $H_{t} / \lambda$ through the value of $K_{l}$ given in Eqs. (6) and (7) so we proceed next to describe how $H_{t}$ is calculated. First, potential flow theory in Ref. [9] predicts that (i) the initial thickness of the lamella is given by

$$
H_{a}=\left(\frac{\sqrt{12}}{3 \pi}\right) R t_{e}^{3 / 2}
$$

and also that (ii) the liquid velocity at the root of the lamella is twice the initial velocity of the edge of the lamella namely, $V_{a}=2 V_{t}$, with $V_{t}$ given in Eq. (4), see the sketch in Fig. 5. However, the real thickness of the root of the lamella, $H_{a}^{+}$, is larger than the prediction given by potential flow theory in Eq. (8) as a consequence of the finite thickness of the boundary layer. Indeed, the thickness $\delta$ of the boundary layer developing at the entrance of the root of the lamella illustrated in Fig. 5 was deduced in Ref. [12] to be

$$
\delta=4.34 \frac{H_{a}}{\sqrt{\operatorname{Re} t_{e}}},
$$

with $t_{e}$ and $H_{a}$ given, respectively, by Eqs. (5) and (8). The thickness $H_{a}^{+}$is then calculated imposing that the value of the flow rate per unit length entering into the lamella is the one given by potential flow theory namely, $2 V_{t} H_{a}$. Then, assuming for simplicity that the velocity varies linearly within the boundary layer,

$$
2 V_{t} H_{a}=2 V_{t}\left(H_{a}^{+}-\delta / 2\right) \Rightarrow H_{a}^{+}=H_{a}+\delta / 2 .
$$

But the diameter $H_{t}$ of the rim limiting the edge of the expanding lamella is even larger than $H_{a}^{+}$ because the liquid flows into the lamella twice as fast as the edge of the lamella, see Fig. 5, and because of the capillary retraction, these facts explaining the experimental result in Ref. [9], where it is found that

$$
H_{t} \approx 3 H_{a} \approx R t_{e}^{3 / 2}
$$

with $t_{e}$ the solution of Eq. (5) and, hence, $K_{l}$ in Eqs. (6) and (7) can now be calculated in terms of the ratio

$$
\frac{H_{t}}{\lambda}=\frac{R}{\lambda} t_{e}^{3 / 2}
$$

and the term at the left of the splash criterion (3) can be fully determined. But, before comparing the experimental results in Fig. 3 with the predictions of Eq. (3), let us first point out that Eqs. (6) 
and (7) for $K_{l}$ were deduced solving the lubrication equations in the wedge region sketched in Fig. 5. Therefore, these equations will only be valid in the continuum aproach namely, when $H_{t}$ in Eq. (11) is larger than the mean free path $\lambda$. Indeed, when the rim thickness is similar to the mean free path of gas molecules i.e., $H_{t} \lesssim \lambda$, it is expected that the lubrication force $F_{L}$ will be zero and, consequently, the drop will not splash. Interestingly, in spite of the mean free path of air molecules is below $100 \mathrm{~nm}$, the thickness of the lamella can be similar to $\lambda$. Indeed, in the limit of low values of the Ohnesorge numbers of interest here, Oh $\ll 1$, the thickness of the boundary layer $\delta$ is smaller than the thickness $H_{a}$ in Eq. (8) and the solution of Eq. (5) can be approximated by $t_{e} \simeq \mathrm{We}^{-2 / 3}$ $[12,25]$. Hence, Eq. (11) reads

$$
H_{t} \approx \frac{\sigma}{\rho V^{2}}
$$

and the ratio $\lambda / H_{t}$ with $H_{t}$ given by Eq. (13) reads

$$
\frac{\lambda}{H_{t}}=\frac{\rho V^{2} \lambda}{\sigma}
$$

Consequently, the parameter $\mathrm{We}_{\lambda}$ defined in Eq. (1) represents a measure of the relative thickness of the lamella with respect to the mean free path of the gas molecules and then equation

$$
\mathrm{We}_{\lambda}=1 \rightarrow V^{\prime} \approx\left(\frac{\sigma}{\rho \lambda}\right)^{1 / 2},
$$

provides with an estimate of the values of the drop impact velocity $V^{\prime}$ for which the thickness of the lamella is close to the mean free path of the gas molecules: $V^{\prime} \approx 20 \mathrm{~m} / \mathrm{s}$ for the case of ethanol and $V^{\prime} \approx 32 \mathrm{~m} / \mathrm{s}$ for the case of water $\left(\sigma=7.2 \times 10^{-2} \mathrm{~N} / \mathrm{m}, \rho=10^{3} \mathrm{~kg} / \mathrm{m}^{3}\right)$.

In view of the discussion above, the value of the coefficient $K_{l}$ in (3) for $\mathrm{We}_{\lambda}>\mathrm{We}_{\lambda 0}$ with $\mathrm{We}_{\lambda 0}$ an order unity constant to be determined from experiments, will be set here to

$$
\text { If } \mathrm{We}_{\lambda}>\mathrm{We}_{\lambda 0} \Rightarrow K_{l}=0 \text {. }
$$

The experimental data in Fig. 3 corresponding to $\mathrm{Oh}_{\lambda}=\mu / \sqrt{\rho \lambda \sigma}=0.91$ as well as the experimental point for the case of water in Visser et al. [5,6] corresponding to $\mathrm{Oh}_{\lambda}=0.46$, are compared in Fig. 6 against the predictions given by Eq. (3) using Eqs. (6) and (7) for $K_{l}$ deduced in Ref. [10] and Eq. (16) for $K_{l}$ deduced here. The agreement between the experimental observations and the theoretical values is rather good when the value of $\mathrm{We}_{\lambda 0}$ in $\mathrm{Eq}$. (16) is set to $\mathrm{We}_{\lambda 0}=0.5$. Notice, however, that some spreading events are seen in the region where splashing is predicted to occur, but the number of these experimental points is quite small when compared with the number of splashing events. The results in Fig. 6 indicate that our theory is able to predict the experimental observations in Fig. 4, namely the spreading-splashing-spreading transition as well as the total absence of splashing, no matter how large the impact velocity is, when droplets are sufficiently small.

Let us point out that the results in Fig. 6 represent just only a part of the full phase diagram. Indeed, for larger impact velocities or smaller drop radii, the boundary layer thickness $\delta$ in Eq. (9) will become of the order of the thickness of the lamella, this happening when

$$
\sqrt{\operatorname{Re} t_{e}} \lesssim 4 \Rightarrow \mathrm{Re}^{1 / 6} \mathrm{Oh}^{2 / 3}>K \equiv \mathrm{We}_{\lambda}>K^{12}(R / \lambda)^{2} \mathrm{Oh}_{\lambda}^{-6},
$$

where we have made use of the approximation $t_{e} \approx \mathrm{We}^{-2 / 3}$. The stripped area in Fig. 7 indicates the region where the condition (17) is satisfied with $K=0.33$, this also being a region where the expressions for the ejection time (5) and for the thickness of the lamella (11) are no longer valid and, instead, the analogous expressions deduced in Ref. [12] should be used in order to predict whether the drop spreads or splashes. These alternative equations for $t_{e}$ and $H_{t}$ involve, however, unknown 


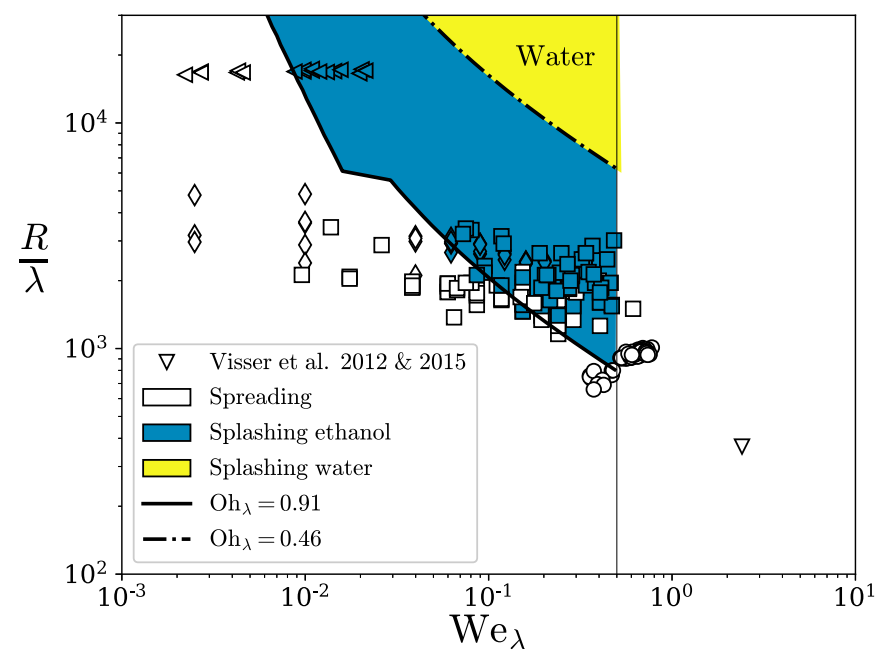

FIG. 6. The boundary separating spreading and splashing conditions obtained experimentally is compared with the theoretical predictions in a $R / \lambda-\mathrm{We}_{\lambda}$ plane, with $\mathrm{We}_{\lambda}=\rho V^{2} \lambda / \sigma$ and $\lambda=68 \times 10^{-9} \mathrm{~m}$. The white region indicates spreading, whereas splashing is represented in blue for the case of ethanol $\left(\mathrm{Oh}_{\lambda}=\mu / \sqrt{\rho \sigma \lambda}=\right.$ $0.91)$ and in yellow for the case of water $\left(\mathrm{Oh}_{\lambda}=0.46\right)$. The thicker continuous line and the dash-dotted black line are used to respectively indicate the predicted spreading-splashing transition for the cases of ethanol (continuous line) and water (dash-dotted line). The vertical thin line indicates $\mathrm{We}_{\lambda}=\mathrm{We}_{\lambda 0}=0.5$. The experimental data point corresponding to the spreading of a water droplet of radius $R=25 \mu \mathrm{m}$ impacting with a velocity of $50 \mathrm{~m} / \mathrm{s}$ on a smooth substrate is taken from Visser et al. [5,6].

constants that need to be determined carrying out new experiments, and this task is beyond the scope of the present study.
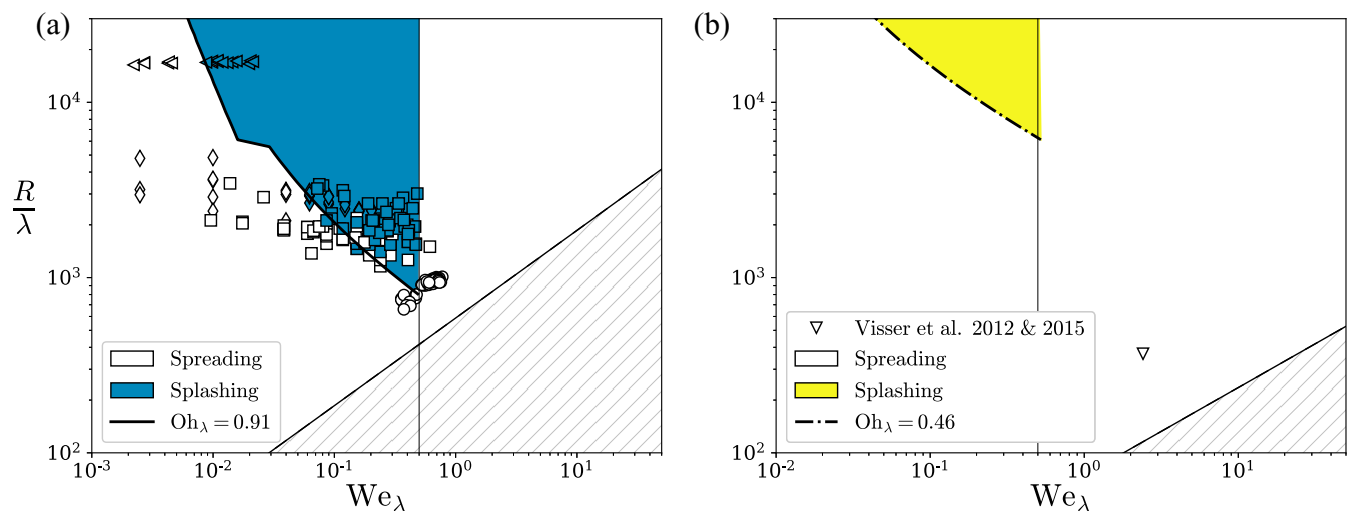

FIG. 7. The condition expressed by Eq. (17) with $K=0.33$ are represented using striped lines in the phase diagrams corresponding to (a) ethanol drops in air at normal atmospheric conditions, for which $\mathrm{Oh}_{\lambda}=0.91$ and (b) water drops in air at normal atmospheric conditions, for which $\mathrm{Oh}_{\lambda}=0.46$. The present theoretical results cannot be applied to predict whether the drop spreads or splashes in the stripped area, which represent conditions under which the thickness of the boundary layer is similar to that of the ejected lamella. The experimental data point corresponding to the spreading of a water droplet of radius $R=25 \mu \mathrm{m}$ impacting with a velocity of $50 \mathrm{~m} / \mathrm{s}$ on a smooth substrate is taken from Visser et al. [5,6]. 


\section{CONCLUSIONS}

To conclude, here we provide with a predictive theory that determines the region in the $R-V$ space in which micron-sized ethanol or water droplets spread over a partially wetting substrate, these being the conditions under which the image resolution of ink-jet printers, is optimal. We have shown that splashing can be suppressed if the drop impact velocity is such that $\mathrm{We}_{\lambda}=\rho V^{2} \lambda / \sigma \gtrsim 0.5$ and also that our theory is capable to predict the spreading-splashing-spreading transition which is observed experimentally as the impact speed is increased when the drop radius is above a certain threshold value. All of these conclusions are valid whenever the thickness of the boundary layer is smaller than the thickness of the lamella: The analysis of the cases for which the thickness of the boundary layer is similar to the thickness of the ejected lamella, corresponding to smaller or faster droplets or to droplets of liquids with viscosities larger than those considered here, is left for a future study.

\section{ACKNOWLEDGMENTS}

This work has been supported by Japan Society for the Promotion of Science (JSPS) KAKENHI Grant Nos. 20H00222 and 20H00223, Institute of Global Innovation Resarch in TUAT, and the Spanish MINECO under Projects No. DPI2017-88201-C3-1-R and No. RED2018-102829-T, partly financed through European funds.

[1] O. A. Basaran, H. Gao, and P. P. Bhat, Nonstandard inkjets, Annu. Rev. Fluid Mech. 45, 85 (2013).

[2] M. Pasandideh-Ford, Y. M. Qiao, S. Chandra, and J. Mostaghimi, Capillary effects during droplet impact on a solid surface, Phys. Fluids 8, 650 (1996).

[3] C. Clanet, C. Beguin, D. Richard, and D. Quéré, Maximal deformation of an impacting drop, J. Fluids Mech. 517, 199 (2004).

[4] I. V. Roisman, Inertia dominated drop collisions. II. An analytical solution of the Navier-Stokes equations for a spreading viscous film, Phys. Fluids 21, 052104 (2009).

[5] C. W. Visser, Y. Tagawa, C. Sun, and D. Lohse, Microdroplet impact at very high velocity, Soft Matter 8 , 10732 (2012).

[6] C. W. Visser, P. E. Frommhold, S. Wildeman, R. Mettin, D. Lohse, and C. Sun, Dynamics of high-speed micro-drop impact: Numerical simulations and experiments at frame-to-frame times below $100 \mathrm{~ns}$, Soft Matter 11, 1708 (2015).

[7] J. M. Gordillo, G. Riboux, and E. S. Quintero, A theory on the spreading of impacting droplets, J. Fluid Mech. 866, 298 (2019).

[8] C. Josserand and S. T. Thoroddsen, Drop impact on a solid surface, Annu. Rev. Fluid Mech. 48, 365 (2016).

[9] G. Riboux and J. M. Gordillo, Experiments of Drops Impacting a Smooth Solid Surface: A Model of the Critical Impact Speed For Drop Splashing, Phys. Rev. Lett. 113, 024507 (2014).

[10] J. M. Gordillo and G. Riboux, A note on the aerodynamic splashing of droplets, J. Fluid Mech. 871, R3 (2019).

[11] L. Xu, W. W. Zhang, and S. R. Nagel, Drop Splashing on a Dry Smooth Surface, Phys. Rev. Lett. 94, 184505 (2005).

[12] G. Riboux and J. M. Gordillo, Boundary layer effects in droplet splashing, Phys. Rev. E 96, 013105 (2017).

[13] A. L. Biance, C. Clanet, and D. Quéré, Leidenfrost drops, Phys. Fluids 15, 1632 (2003).

[14] H. J. Staat, T. Tran, B. Geerdink, G. Riboux, C. Sun, J. M. Gordillo, and D. Lohse, Phase diagram for droplet impact on superheated surfaces, J. Fluid Mech. 779, R3 (2015).

[15] T. Tran, H. J. J. Staat, A. Prosperetti, C. Sun, and D. Lohse, Drop Impact on Superheated Surfaces, Phys. Rev. Lett. 108, 036101 (2012). 
[16] M. Shirota, M. A. J. Van Limbeek, C. Sun, A. Prosperetti, and D. Lohse, Dynamic Leidenfrost Effect: Relevant Time and Length Scales, Phys. Rev. Lett. 116, 064501 (2016).

[17] D. Richard and D. Quéré, Bouncing water drops, Europhys. Lett. 50, 769 (2000).

[18] D. Richard, C. Clanet, and D. Quéré, Contact time of a bouncing drop, Nature 417, 811 (2002).

[19] J. C. Bird, R. Dhiman, H. M. Kwon, and K. K. Varanasi, Reducing the contact time of a bouncing drop, Nature 503, 385 (2013).

[20] J. De Ruiter, R. Lagraauw, D. Van Den Ende, and F. Mugele, Wettability-independent bouncing on flat surfaces mediated by thin air films, Nat. Phys. 11, 48 (2015).

[21] E. S. Quintero, G. Riboux, and J. M. Gordillo, Splashing of droplets impacting superhydrophobic substrates, J. Fluid Mech. 870, 175 (2019).

[22] H. Onuki, Y. Oi, and Y. Tagawa, Microjet Generator for Highly Viscous Fluids, Phys. Rev. Applied 9 , 014035 (2018).

[23] J. M. Gordillo, H. Onuki, and Y. Tagawa, Impulsive generation of jets by flow focusing, J. Fluid Mech. 894, 1 (2020).

[24] J. Hao, J. Lu, L. Lee, Z. Wu, G. Hu, and J. M. Floryan, Droplet Splashing on an Inclined Surface, Phys. Rev. Lett. 122, 054501 (2019).

[25] G. Riboux and J. M. Gordillo, Maximum drop radius and critical Weber number for splashing in the dynamical Leidenfrost regime, J. Fluid Mech. 803, 516 (2016). 